\title{
Correlation of prefrontal activity measured by near-infrared spectroscopy (NIRS) with mood, BDNF genotype and serum BDNF level in healthy individuals
}

\author{
Daisuke Matsuzawa ${ }^{1 *}$, Kotaro Takeda ${ }^{2,3}$, Hiroyuki Ohtsuka ${ }^{1}$, Jun Takasugi ${ }^{1}$, Takashi Watanabe ${ }^{1}$, \\ Junko Maeda $^{1}$, Saeka Nagakubo ${ }^{1}$, Chihiro Sutoh ${ }^{1}$, Ichiro Shimoyama ${ }^{4}, K^{1}$ Nakazawa ${ }^{1}$, Eiji Shimizu $^{1}$ \\ ${ }^{1}$ Department of Cognitive Behavior Physiology, Graduate School of Medicine, Chiba University, Chiba, Japan \\ ${ }^{2}$ Clinical Research Center, National Hospital Organization Murayama Medical Center, Tokyo, Japan \\ ${ }^{3}$ Department of Motor Control and Rehabilitation, ATR Computational Neuroscience Laboratories, Kyoto, Japan \\ ${ }^{4}$ Section for Human Neurophysiology, Frontier Medical Engineering, Chiba University, Chiba, Japan

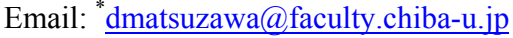

Received 25 April 2012; revised 31 May 2012; accepted 9 June 2012

\begin{abstract}
Depression has been known to reduce the prefrontal activity associated with the execution of certain cognitive tasks, although whether a temporarily depressed or anxious mood in healthy individuals affects the prefrontal blood oxygen level during cognitive tasks is unknown. Combining the measurement of prefrontal activity with near-infrared spectroscopy (NIRS) and the two cognitive tasks, namely the letter version of the verbal fluency test (VFT-I) and the Stroop test, we measured the effect of a depressed or anxious mood and gender on the changes in the prefrontal oxygenated hemoglobin (Oxy-Hb) levels during those cognitive tests in healthy individuals. Depressed mood or anxious mood was assessed by the Hospital Anxiety and Depression Scale (HADS). Thereby we aimed to explore the possibility of NIRS measurement for detecting the early subclinical manifestation of major depression. Moreover, we examined the possible relationships between prefrontal activation and the functional Val66Met polymorphisms of the brain derived neurotropic factor (BDNF) gene and serum BDNF level. As a result, the increased prefrontal Oxy-Hb levels during cognitive tasks were significantly correlated with the severity of depressed mood in males. The course of the prefrontal Oxy-Hb increase was different depending on the cognitive tasks, i.e., the VFT-I or the Stroop test, in both genders. Correlations of BDNF genotype and serum BDNF level with the prefrontal Oxy-Hb levels during those cognitive tasks were negative. Our results suggest that the early subclinical manifestation of depressed mood in males might be detected by the
\end{abstract}

"Corresponding author.
NIRS measurement, which is not correlated with the individual properties of BDNF.

Keywords: Near-Infrared Spectroscopy (NIRS); Depression; Anxiety; Brain Derived Neurotropic Factor (BDNF)

\section{INTRODUCTION}

Depression is a common psychiatric disease and results in considerable social-economic burdens due to loss of productivity [1-3]. Thus, the prevention of depression with early detection and intervention is critically important for those exposed to chronic stress because of its potential to induce depression in some individuals $[4,5]$.

Near-infrared spectroscopy (NIRS) is a non-invasive method of measuring the relative change in the concentrations of oxygenated and deoxygenated hemoglobin $(\Delta$ [Oxy-Hb]) and $\Delta[\mathrm{DeOxy}-\mathrm{Hb}]$, respectively), which are closely correlated to the neural activity of the brain [6]. Using this technique, altered prefrontal activation has been reported in various psychiatric diseases such as schizophrenia, major depression, bipolar disorder and anxiety disorder [7]. In the case of major depression, a reduced increase of the dorsolateral prefrontal $\mathrm{Oxy}-\mathrm{Hb}$ level compared to healthy controls during the letter version of the Verbal Fluency Test (VFT-1-1) as determined by the measurement of NIRS has been reported repeatedly [8-10]. However, it has not been clarified whether a temporarily depressed or anxious mood in healthy individuals affects the frontal blood oxygen level during cognitive tasks. Given that one of the main symptoms of major depression is depressive mood, it is a matter of concern whether prefrontal activations could be affected by such a mood. In addition, several lines of evidence 
have shown that there exist gender differences in the prevalence, comorbidity, and symptom presentation pattern in major depression [11], thus, early manifestations of the disease might be different between the genders, and these differences might be observed in the altered prefrontal activation measured by NIRS.

Brain derived neurotrophic factor (BDNF), the most abundant neurotrophic factor in the human brain, plays important roles in the survival, development, and proliferation of the central and peripheral neurons [12,13]. A functional polymorphism of the BDNF gene, an amino acid substitution of a valine for a methionine at codon 66 (Val66Met) as a single nucleotide polymorphism (SNP), has been suggested to be associated with the performance of episodic memory in schizophrenia patients [14] as well as healthy control subjects $[14,15]$, and with the performance of prefrontal cognitive tasks in bipolar disorder [16]. In addition, accumulating evidence suggests that serum BDNF level, which likely reflects brain BDNF level, is altered in mood disorders such as depression. Serum BDNF level has been reported to be associated with depression severity [17] and has been observed to be increased after antidepressant treatment in depressed patients [18-20]. One of the personally traits, neuroticism in the NEO-five factor inventory (NEO-FFI), which is considered to be a marker of depression, appeared to have a negative correlation with serum BDNF level in healthy humans [21]. Taken together, these findings suggest that prefrontal activation is potentially correlated with each individual's BDNF profile, but to date no NIRS study has been conducted to assess this potential correlation.

In the present study, we examined the correlation of changes in the prefrontal $\mathrm{Oxy}-\mathrm{Hb}$ levels measured by NIRS during cognitive tasks with depressed or anxious mood, gender, Val66Met polymorphisms of the BDNF gene and serum BDNF level in basically healthy individuals. Thereby we aimed to explore the possibility of using NIRS measurement to detect early subclinical manifestations of major depression or anxiety disorder.

Part of the entire journals, and not as an independent document. Please do not revise any of the current designations.

\section{MATERIALS AND METHODS}

\subsection{Subjects and Blood Samples}

The research was performed after the study was approved by the ethics committee of Chiba University Graduate School of Medicine and Chiba Prefectural College of Allied Medical Science. All of the subjects were the students of Chiba Prefectural College of Allied Medical Science. Subjects were well explained about the experiments, and written informed consent was obtained.
Thirty eight healthy volunteers (female $=17$, male $=20$, mean age $=22.6 \pm$ SD 3.7, range $=19-31)$ were enrolled in the NIRS study. None of them had past history of psychotic disorders of the DSM-IV axis I or II, neurological disorders, drug dependence, and any major physical illness. One female subject was excluded from the following analyses because of developing depression which needed medical service three months after the NIRS measurements. All except one male subject were righthanded. Whole blood samples for extracting DNA and serum samples for BDNF measurement were collected from 30 of those 37 subjects $($ female $=13$, male $=17$ ) between 11:00-12:00 AM and stored at $-80^{\circ} \mathrm{C}$.

\subsection{Assessment of Metal Status with HADS}

Hospital Anxiety and Depression Scale (HADS) was used to assess subjects' mental status. HADS is a selfreport screening scale that was developed to indicate the possible presence of anxiety and depression states in hospital and medical outpatient clinic settings. HADS consists of a seven-item anxiety subscale and a sevenitem depression subscale. Each item is scored on a four-point scale, giving maximum subscale scores of 21 for depression and anxiety, respectively. Cut-off scores were recommended as $\geq 8$ for possible anxiety or depression in the original study [22]. Self-assessment of HADS was conducted blind to the experimenter of NIRS (H.O. and J.T.), BDNF measurement and genotyping (D.M.). In addition, analysis of HADS score was performed after all the other results were obtained in order to keep subjects' mental status blind to those measurements. Some of them reached $\geq 8$ in the scores of depression and anxiety subscales of HADS, but it was because the study was done right before the semester final examination, and none of them met the criteria of any mental illnesses in DSM-IV [23] when the NIRS measurement and blood collection were conducted. Thus, their mental statuses were assumed as healthy overall, even though temporal depressed or anxiety moods were existed.

\subsection{NIRS Study}

\subsubsection{NIRS Recordings}

NIRS recordings were performed using a multichannel optical topography system (FOIRE-3000, Shimadzu Co., Japan). Eleven light-emission and ten light-detection probes were arranged in a $3 \times 7$ rectangular lattice. Each recording channel consisted of one light-emission probe and one light-detection probe located $3 \mathrm{~cm}$ away from each other. A head shell that mounted the 21 probes for 32 recording channels was placed securely on the scalp overlying mainly the prefrontal area. The light-detection probe between channels No.3 and No.4 was located on Fz of the international 10 - 20 system (Figure 1(a)). 
(a)
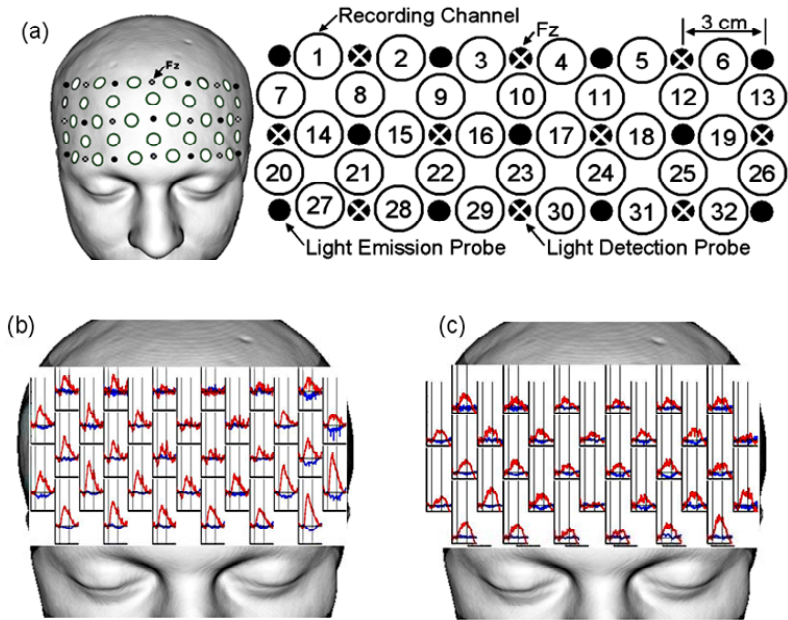

Figure 1. Probe setting and typical courses of the Oxy- and DeOxy-Hb change during the tasks. (a) 21 probes for 32 recording channels were placed securely on the scalp overlying mainly the prefrontal area. The light-detection probe between channels No.3 and No.4 was located on Fz of the international 10 - 20 system. Eleven light-emission and ten light-detection probes were arranged in a $3 \times 7$ rectangular lattice. Each recording channel consisted of one light-emission probe and one light-detection probe located $3 \mathrm{~cm}$ away from each other; (b), (c) Typical courses of the Oxy-Hb (red line) and DeOxy-Hb (blue line) change during the two tasks, VFT-1 (b) and Stroop test (c).

\subsubsection{Cognitive Tasks for NIRS Recording}

Prefrontal activities were recorded during the letter version of verbal fluency test (VFT-1) and Stroop tasks. For NIRS recording, each cognitive task was consisted of a $30 \mathrm{~s}$ pre-task baseline period, a $60 \mathrm{~s}$ task period, and a 60 s post-task baseline period. Instruction cues for each task were displayed on a 22 -inch monitor connected to a laptop personal computer placed approximately 1 meter in front from the subject who was sitting on a comfortable chair.

The VFT-1 was carried out according to the previous studies $[10,24]$. In briefly, during the task period, subjects were instructed to produce orally as many words that began with the cue as possible. During the task period, Three Japanese letters (/a/, /ka/, and $/ \mathrm{sa} /)$ were presented on the display as cues in turn every $20 \mathrm{~s}$. During the pre- and post-task baseline periods, the subjects were instructed to repeat the syllables $/ \mathrm{a} /, / \mathrm{i} /, / \mathrm{u} /, / \mathrm{e} /$, and $/ \mathrm{o} /$.

To assess the Stroop effect [25], a list of color names (blue, yellow, red, and green, written in Japanese Kanji characters), which were printed in a color not denoted by the name, was presented during the task period, and a list of color dots (blue, yellow, red, and green) was presented during the pre- and post-task baseline periods. A set of 24 dots/words was presented consecutively on the display. The subjects were instructed to name the ink color in which the dots/words were presented as quickly as pos- sible and explicitly not to read the words. The investigator recorded the number of correct and incorrect verbal responses.

\subsubsection{NIRS Data Analyses}

Three near-infrared laser beams (wavelengths at 780, 805, and $830 \mathrm{~nm}$ ) were emitted, and reflectance beams sampled at a $175-\mathrm{ms}$ sampling interval were used to calculate $\Delta[\mathrm{Oxy}-\mathrm{Hb}]$ and $\Delta[\mathrm{DeOxy}-\mathrm{Hb}]$. The linear trends of the continuous $\Delta[\mathrm{Oxy}-\mathrm{Hb}]$ and $\Delta[\mathrm{DeOxy}-\mathrm{Hb}]$ fluctuations were removed and smoothed with a five-point moving average. In the present study, we mainly report the $\Delta$ [Oxy- $\mathrm{Hb}]$ results because it may be the most sensitive parameter among hemodynamic responses [26]. Many previous NIRS studies calculated a z score in each recording channel for comparison among subjects or among recording channels [27-29], therefore, we also used the $\mathrm{z}$ score for comparison. In the present study, the $\mathrm{z}$ score was calculated in each recording channel as follows: the mean $\Delta[\mathrm{Oxy}-\mathrm{Hb}]$ value during the task period (30 - $60 \mathrm{~s}$ after task onset) versus that during a 30-s pre-task baseline period was divided by the standard deviation (SD) of $\Delta[\mathrm{Oxy}-\mathrm{Hb}]$ during the pre-task period. The signal processing was performed using MATLAB 7.5.0 (MathWorks, Natick, MA, USA). The significance level of $\Delta[\mathrm{Oxy}-\mathrm{Hb}]$ was set at $\mathrm{z}>3.163$, which reached the two-sided Bonferroni corrected significance level ( $p$ $<-0.05)$. This calculation method for the significance level is similar to that used in previous NIRS studies [28, 29]. The mean $z$ score of $\Delta[\mathrm{Oxy}-\mathrm{Hb}]$ over all channels was analyzed to measure the signal intensity, and the number of channels with a significant $\Delta[\mathrm{Oxy}-\mathrm{Hb}]$ was analyzed to measure the extent of cerebral activation. Typical courses of the change in Oxy- and DeOxy-Hb during the two tasks, namely the VFT-1 and the Stroop test, are shown in Figures 1(b) and (c), respectively.

\subsection{Genotyping}

The genomic DNA was extracted from peripheral leukocytes by standard procedures. Polymerase chain reaction (PCR) and the PCR-based restriction fragment length polymorphism (RFLP) assay were performed to genotype the DNA sequence variants of the BDNF gene as reported previously [30]. The primer sequences were forward: 5-GGTGAGAAGAGTGATGACCA-3 and reverse: 5-GCCAGCCAATTCTCTCTTTTT-3. The PCR products were digested at $37^{\circ} \mathrm{C}$ with restriction enzyme PmaC I (Takara Shuzo Ltd., Kyoto, Japan), followed by $2 \%$ agarose gel-electrophoresis with ethidium bromide staining. After testing the Hardy-Weinberg equilibrium for genotype frequencies (Val/Val, Val/Met and Met/Met), subjects were divided by two groups, Val/Val and Met carrier (Val/Met and Met/Met) group. 


\subsection{Serum BDNF Measurements}

Serum BDNF levels were measured using the BDNF Emax Immunoassay System kit (Promega, Madison, WI) according to the manufacturer's instructions. Briefly, 96well plates were coated with anti-BDNF monoclonal antibody and incubated at $4^{\circ} \mathrm{C}$ for 18 hours. The plates were incubated in a blocking buffer for 1 hour at room temperature. The samples and BDNF standards were maintained at room temperature under conditions of shaking for 2 hours, followed by washing with the appropriate washing buffer. The plates were incubated with antihuman BDNF polyclonal antibody at room temperature for 2 hours, washed, and incubated with anti-IgY antibody conjugated to horseradish peroxidase for 1 hour at room temperature. The plates were incubated in peroxidase substrate and tetramethylbenzidine solution to produce a color reaction. The reaction was stopped with $1 \mathrm{~mol} / \mathrm{L}$ hydrochloric acid, the absorbance at $450 \mathrm{~nm}$ was measured with an Emax automated microplate reader (Molecular Devices, Tokyo, Japan).

\subsection{Statistical Analysis}

All the calculations were performed with SPSS software (SPSS version 17.0J, Tokyo, Japan). Student's t-test (two-tailed) was employed for each comparison of NIRS measurements and BDNF levels between the genders, and BDNF Val66Met genotypes. Spearman's correlation coefficients were examined to identify the correlation of the intensity of the Oxy-Hb increase during the tasks with the genders, and with the serum BDNF level. The Hardy-Weinberg equilibrium for genotype frequencies was calculated using chi-square test. A value of $p<0.05$ was used as the standard for statistical significance in all the analyses.

\section{RESULTS}

\subsection{Scores of HADS and Cognitive Tasks between the Gender}

The mean HADS scores and the cognitive task performance of males and females were summarized in Table 1. As is shown, none of the valuables was significantly different between the genders..

\subsection{Gender Difference in the Prefrontal Activity}

Table 2 shows the gender difference in the prefrontal activity. As shown, the mean $\mathrm{z}$ scores of $\Delta[\mathrm{Oxy}-\mathrm{Hb}]$ over all channels during the VFT-1 were $6.52 \pm 4.8$ in males and $2.85 \pm 2.1$ in females. These results were significantly different between the genders $(t=2.95, p<0.007)$. In addition, the mean number of channels with a significant increase in $\Delta[\mathrm{Oxy}-\mathrm{Hb}]$ during the VFT-1 was significantly different between the genders, $19.1 \pm 8.1$ in
Table 1. HADS and the cognitive test performances.

\begin{tabular}{cccc}
\hline & Males & Females & $\mathrm{p}^{*}$ \\
\hline HADS score (mean score \pm SD) & & \\
depression & $3.0 \pm 2.6$ & $3.1 \pm 3.0$ & n.s. \\
anxiety & $5.0 \pm 3.6$ & $4.3 \pm 2.9$ & n.s. \\
Cognitive test performance (mean score \pm SD) & \\
words production in the VFT-1 & $14.1 \pm 4.1$ & $16.3 \pm 4.4$ & n.s. \\
correct words in the Stroop & $73.8 \pm 16.8$ & $75.2 \pm 11.5$ & n.s. \\
incorrect words in the Stroop & $2.1 \pm 2.0$ & $2.6 \pm 1.7$ & n.s. \\
\hline
\end{tabular}

t-test. HADS: Hospital Anxiety and Depression Scale; VFT-1: Letter version of the verbal fluency test; Stroop: Stroop test.

Table 2. Effect of gender on the prefrontal activity.

\begin{tabular}{rccc}
\hline & \multicolumn{3}{c}{ Gender (n) } \\
\hline [Oxy-Hb] during VFT-1 & Males (9) & Females (21) & $\mathrm{p}^{3)}$ \\
$\begin{array}{c}\mathrm{z} \text { scores over all channels }{ }^{1)} \\
\text { the number of channels, } \\
\mathrm{z}>3.163^{2)}\end{array}$ & $6.52 \pm 4.8$ & $2.85 \pm 2.1$ & 0.007 \\
$\begin{array}{c}\Delta[\text { Oxy-Hb] during Stroop } \\
\text { z scores over all channels }{ }^{1)} \\
\text { the number of channels, } \\
\mathrm{z}>3.163^{2)}\end{array}$ & $1.73 \pm 1.8$ & $10.2 \pm 7.5$ & 0.003 \\
\hline
\end{tabular}

${ }^{11}$ Mean $\mathrm{z}$ score $\pm \mathrm{SD}$ over all channels; ${ }^{2)}$ Mean number of channels $\pm \mathrm{SD}$ with significant increase $(z>3.163)$ during the task ${ }^{3} t$-test.

males and $10.2 \pm 7.5$ in females $(t=3.24, p=0.003)$.

Thus, prefrontal activation during the VFT-1 was significantly more intense and broader in males than females.

In contrast, the mean $\mathrm{z}$ scores of $\Delta[\mathrm{Oxy}-\mathrm{Hb}]$ over all channels during the Stroop test, $1.73 \pm 1.8$ in males and $1.67 \pm 2.2$ in females $(t=0.092, p=0.93)$, were not significantly different between the genders, and the mean number of channels with a significant increase in $\Delta$ [Oxy-Hb] during the Stroop test, $8.75 \pm 7.1$ in males and $9.18 \pm 8.0(\mathrm{t}=-0.17, \mathrm{p}=0.87)$, was not significantly different between the genders.

\subsection{Correlation of Prefrontal Oxy-Hb Increase with HADS Scores}

Spearman's correlation coefficients revealed a significant correlation between the depression scores of HADS and the mean $\mathrm{z}$ score of $\Delta[\mathrm{Oxy}-\mathrm{Hb}]$ over all channels during both the VFT-1 $(\rho=0.57, \mathrm{p}=0.009)$ and the Stroop test $(\rho=0.55, \mathbf{p}=0.012)$ in males (Figures 2(a) and (b)). In contrast, in females (Figures 2(a) and (b)), the depression scores of HADS were not correlated with the mean $\mathrm{z}$ score of $\Delta[\mathrm{Oxy}-\mathrm{Hb}]$ over all channels during both the VFT-1 $(\rho=-0.29, \mathrm{p}=0.247)$ and the Stroop test $(\mathrm{r}=$ 


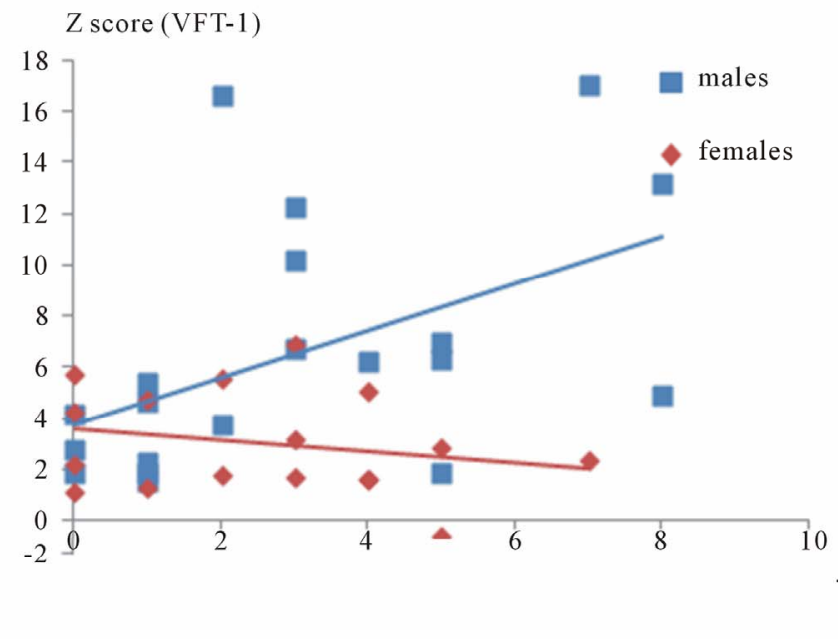

HADS-Depression score

(a)

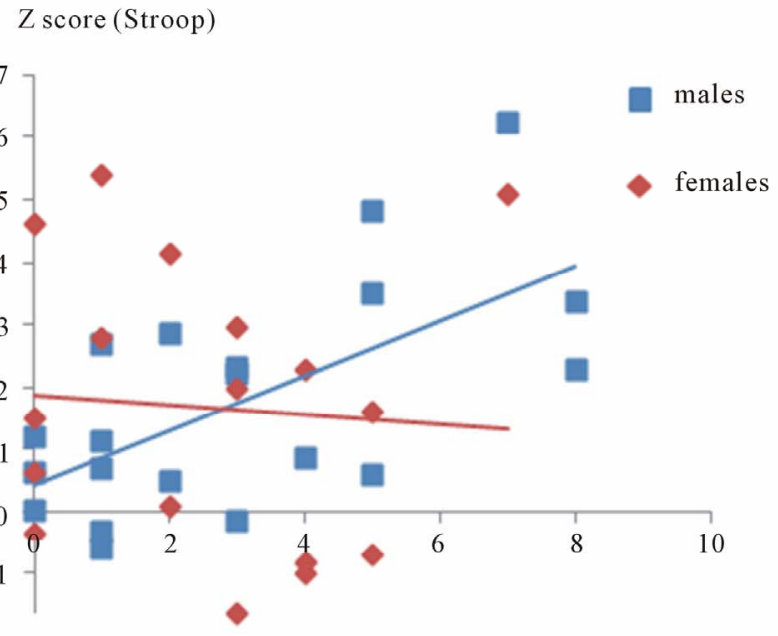

HADS-Depression score

(b)

Figure 2. Correlation of the intensity of the prefrontal Oxy-Hb increase with HADS depression scores in both of the genders. (a) A significant correlation between the depression scores of HADS and the mean z score of $\Delta[\mathrm{Oxy}-\mathrm{Hb}]$ over all channels during the VFT-1 was observed in the males $(\rho=0.57, \mathrm{p}=0.009)$ but not in the females $(\rho=-0.29, \mathrm{p}=0.247)$; (b) A significant correlation between the depression scores of HADS and the mean z score of $\Delta[\mathrm{Oxy}-\mathrm{Hb}]$ over all channels during the Stroop test was observed in the males $(\rho=0.55, \mathrm{p}=0.012)$ but not in the females $(\rho=-0.24, \mathrm{p}=0.340)$.

$-0.24, p=0.340)$. In both genders, the anxiety scores of HADS were not significantly correlated with the mean $\mathrm{z}$ score of $\Delta[\mathrm{Oxy}-\mathrm{Hb}]$ over all channels during both the VFT-1 (males, $\rho=0.20, \mathrm{p}=0.402$, females, $\rho=-0.167, \mathrm{p}$ $=0.507$ ) and the Stroop test (males, $\rho=0.169, \mathrm{p}=0.477$, females, $\rho=0.018, \mathrm{p}=0.945$ ).

\subsection{Effect of BDNF Val66Met Polymorphism on the Prefrontal Activity}

Genotype distribution was in Hardy-Weinberg equilibrium $\left(\chi^{2}=1.15, \mathrm{df}=1, \mathrm{p}=0.564\right)$. As shown in Table 2 , 9 subjects were Val/Val type and 21 subjects were Met carriers (17 Val/Met subjects and 4 Met/Met subjects). There was no significant difference between them in the mean $\mathrm{z}$ score of $\Delta[\mathrm{Oxy}-\mathrm{Hb}]$ over all channels or in the mean number of channels with a significant increase in $\Delta[\mathrm{Oxy}-\mathrm{Hb}]$ during both of the tasks (Table 3). The word "data" is plural, not singular.

\subsection{Correlation between the Serum BDNF Level and the Prefrontal Activity}

Spearman's correlation coefficients revealed no correlation between the serum BDNF level and the mean $\mathrm{z}$ score of $\Delta[\mathrm{Oxy}-\mathrm{Hb}]$ over all channels during both the VFT-1 $(\rho=-0.101, \mathrm{p}=0.595)$ and the Stroop test $(\rho=$ $-0.194, \mathrm{p}=0.305)$. In addition, there was no difference in the comparisons of mean serum BDNF level $(\mu \mathrm{g} / \mathrm{ml})$ between the genders $($ Male $=15.24 \pm 3.9$, Female $=$ $14.31 \pm 3.6$, mean $\pm \mathrm{SD}$ ) and between the genotypes
$(\mathrm{Val} / \mathrm{Val}=14.81 \pm 3.3$, Met carriers $=14.85 \pm 4.0$, mean $\pm \mathrm{SD})$.

\section{DISCUSSION}

The major findings of this study were as follows. First, the increased Oxy-Hb levels in the prefrontal lobe during the cognitive tasks (VFT-1 and Stroop test) were significantly correlated with the severity of depressed mood only in males. Second, the course of the Oxy-Hb increase in the frontal lobe was different depending on the cognitive task, i.e. VFT-1 or Stroop task, in both genders. Finally, the correlations of the BDNF genotype and serum BDNF level with the Oxy-Hb levels in the prefrontal lobe during cognitive tasks were negative. To the best of

Table 3. Effect of BDNF Val66Met polymorphism on the pre frontal activity.

\begin{tabular}{|c|c|c|c|}
\hline \multicolumn{4}{|c|}{ Genotype (n) } \\
\hline [Oxy-Hb] during FT-1 & Val/Val (9) & Met carrier (21) & $\mathrm{p}^{3)}$ \\
\hline z scores over all channels ${ }^{1)}$ & $4.86 \pm 3.3$ & $5.00 \pm 4.7$ & n.s \\
\hline $\begin{array}{l}\text { the number of channels, } \\
\qquad \mathrm{z}>3.163^{2)}\end{array}$ & $15.7 \pm 9.0$ & $15.0 \pm 9.3$ & n.s. \\
\hline \multicolumn{4}{|l|}{ [Oxy-Hb] during Stroop } \\
\hline z scores over all channels ${ }^{1)}$ & $1.98 \pm 1.8$ & $1.41 \pm 2.2$ & n.s. \\
\hline $\begin{array}{l}\text { the number of channels, } \\
\qquad \mathrm{z}>3.163^{2)}\end{array}$ & $10.3 \pm 6.7$ & $7.81 \pm 7.9$ & n.s. \\
\hline
\end{tabular}

${ }^{1)}$ Mean $\mathrm{z}$ score $\pm \mathrm{SD}$ over all channels; ${ }^{2)}$ Mean number of channels $\pm \mathrm{SD}$ with significant increase $(z>3.163)$ during the task ${ }^{3}$ t-test. 
our knowledge, this is the first study to report the correlation of mood, BDNF genotype and BDNF level with the prefrontal activity as measured by NIRS in healthy individuals.

\subsection{Correlation of the Increased Prefrontal Oxy-Hb Levels with Depressed Mood in Males}

Even a healthy person sometimes falls into a temporary mood of depression or anxiety due to the stress or events of daily life, and if such a mood is long-lasting it can lead to disability. Figure 2 shows that the depression scores of HADS were significantly correlated with the mean z-scores of Oxy-Hb level during the VFT-1 ( $\rho=$ $0.57, \mathrm{p}=0.009)$ and Stroop $(\rho=0.55, \mathrm{p}=0.012)$ tasks in males. The results suggest that, in males, the heavier the depressed mood, the greater the blood oxygen level required in the prefrontal lobe for the completion of certain cognitive tasks. These were rather unexpected results considering the results of the previous NIRS studies on major depression, in which the increase of the $\mathrm{Oxy}-\mathrm{Hb}$ levels of the prefrontal lobe during the VFT-1 were reduced [8-10]. The major difference between our study and those previous studies was that the subjects of our study were basically healthy individuals and did not meet any criteria of DSM-IV. In healthy individuals, intrasubject reproducibility of the Oxy-Hb increase during the VFT-1 over time as measured by NIRS has been reported $[31,32]$. However, the mental statuses of the healthy individuals in those studies were "within the healthy range", thus, it has not been clarified whether a temporary depressed or anxious mood in healthy individuals would affect the prefrontal $\mathrm{Oxy}-\mathrm{Hb}$ increase during cognitive tasks. Two possibilities should be considered to explain the inconsistency. First, baseline blood perfusion does not change along with a temporarily depressed mood, but the prefrontal lobe of men with depressed mood does not work efficiently during cognitive tasks, and that leads to the high Oxy-Hb levels as seen in Figure 2. It is well known that cortical activation is affected by the complexity and difficulty of the experimental task, as difficult tasks demand more cortical activation than easier tasks $[33,34]$. On the other hand, the scores of the VFT-1 and the Stroop task were not correlated with the HADS scores in either gender (data not shown). Taken together, these findings suggest that even in a depressed mood, males can do the tasks at the expected level by supplying the required brain area with a high Oxy-Hb level. However, when they fall into a serious depressive disorder that meets clinical criteria such as DSM-IV (American Psychiatric Association, 1994), they might no longer be able to supply sufficient $\mathrm{Oxy}-\mathrm{Hb}$ to the required brain area, and this might cause their performance level to drop. Second, depressed mood might cause the baseline $\mathrm{Oxy}-\mathrm{Hb}$ levels to decrease in males, but the Oxy-Hb level might rise substantially higher than in males in a normal mood status when cognitive tasks are undertaken. It is important to note that reduced global cerebral blood flow $(\mathrm{CBF})$ or metabolism has been reported in major depression, but the regional reduction and its correlation with mood remain to be determined [35-37]. Currently, we cannot strictly differentiate those two possibilities since the changes in Oxy- or Deoxy-Hb level measured by NIRS are not absolute but relative changes.

\subsection{Gender Difference in the Increase of Prefrontal Oxy-Hb Level during the Tasks}

There was marked gender difference in the Oxy-Hb level change during the VFT-1. The mean relative increase of the Oxy-Hb level and the number of probes showing a highly increased $\mathrm{Oxy}-\mathrm{Hb}$ level $(\mathrm{z}>3.163)$ were both significantly greater in males than in females (Table 2). To date, a gender difference in prefrontal Oxy-Hb change during the VFT-1 in healthy subjects has been studied in two studies. In one study, female subjects showed less increased Oxy-Hb levels during the VFT-1 than male subjects [38], and in another they showed no change in Oxy-Hb levels compared to males during the VFT-1 [39]. One of the possible reasons for this difference is the difference in experimental design. As indicated by Herrmann et al. [39], they used a passive resting baseline condition before and after the task, whereas Kameyama et al. [38] used an active baseline condition in which the subjects repeated a train of syllables. The procedure of the VFT-1 in our study was almost the same as that in Kameyama et al. [38], and our results seemed to confirm their results. In contrast, this gender difference was not found during the Stroop test. As in our results, no clear gender difference was observed in the previous NIRS studies involving the Stroop test in healthy individuals [40-42]. One possibility is that, during the Stroop test, the medial part of the brain region such as the anterior cingulate cortex might be more involved than the dorsolateral prefrontal region $[43,44]$. Also, Kameyama et al. [38] discussed the gender difference by citing previous reports in which resting global CBF was observed to be higher in female subjects than in male subjects $[45,46]$, thus, increased baseline perfusion could result in decreased activation due to the ceiling effect [38]. One of the limitations of our study was that we did not exchange the order of the two tasks (the VFT-l was always first), and so we cannot exclude the possibility that the order effect influenced our results. If a transient increase of prefrontal perfusion during the VFT-l pushed up the baseline perfusion in males, it is possible that the following increase of Oxy-Hb level during the Stroop test 
was saturated. Finally, it is possible that females use a broader brain area outside the prefrontal area during the VFT-1 than males do, so that the increase of the Oxy-Hb level seemed lower in females. To the best of our knowledge, no clear evidence for this latter explanation has been reported, but it has been reported that the brain activation during linguistic tasks in males might be more lateralized than in females [47], which implies that brain activation could be different between the genders according to the task.

\subsection{Correlation of the Prefrontal Oxy-Hb Increase with BDNF}

In the present study, the correlations of BDNF polymerphism, Val66Met, and serum BDNF level with the $\mathrm{Oxy}-\mathrm{Hb}$ levels in the prefrontal lobe during cognitive tasks were negative in healthy subjects. Accumulating evidence suggests that the BDNF Val66Met polymerphism affects cognitive performance. In previous studies, subjects with Met carriers scored lower in the cognitive tasks than subjects with Val/Val genotypes, and relevance has been found in healthy subjects $[14,15]$ as well as in patients with mental illness such as schizophrenia [15] and bipolar disorder [16]. One of the possible reasons for our negative result is that the performance of the episodic memory task associated with the BDNF genotypes in the previous studies with fMRI reflected hippocampal activation rather than prefrontal activation $[15,48]$. Thus the effect of the Val66Met polymorphism of BDNF might be difficult to detect by NIRS measurement. Another is that the relevance between the BDNF genotype and the prefrontal activation might be illness specific. Scores on the Wisconsin Card Sorting Test (WCST), which activates the dorsolateral prefrontal cortex, was reported to be associated with the BDNF variance in bipolar disorder but not in schizophrenia or in healthy controls [49]. In addition, it should be considered that no difference in cognitive function according to the BDNF genotype has yet been reported in a healthy Japanese population, and the distribution of the BDNF genotype shows clear ethnic differences [50]. Thus, our results might be different from those involving subjects of other ethnicities. To date, there is only one study that suggested a correlation between functional genotype variation and NIRS measurement. By using the 52-channel NIRS with the VFT-1, Takizawa et al. (2009) suggested that a functional SNP of catechol-O-methyltransferase (COMT), Met108/158Val, might affect the prefrontal Oxy-Hb level in subjects with schizophrenia, although relevance was not seen in healthy controls [51]. Serum BDNF level has been indicated to be correlated with the severity of major depression [30] and with treatment response [18-20], but there has been no report that investigated the correlation with prefrontal activity measured by NIRS. Our results suggest that there was no correlation between serum BDNF level and prefrontal activity in healthy individuals. Our results also suggest that, in healthy subjects, the BDNF level is neither affected by temporal depressed mood and/or anxiety nor correlated with the Oxy-Hb level of the prefrontal lobe measured by NIRS. Since neither of our negative results regarding the relevance of the Oxy-Hb levels measured by NIRS to BDNF genotype and serum BDNF level disproves that the relevance might be altered in those who suffer from mental illnesses such as mood disorders, anxiety disorders, or schizophrenia, further study with such patients would be of great interest. Moreover, it should be considered a limitation of our study that we could not collect the blood samples from all of the subjects recruited in the NIRS study. Thus, our results should be considered preliminary.

\section{CONCLUSION}

In applying NIRS measurement to basically healthy individuals, it was found that the increase of the prefrontal Oxy-Hb level during cognitive tasks (VFT-1 and Stroop test) was significantly correlated with depressed mood only in males. In addition, the course of the Oxy-Hb increase in the prefrontal lobe was different depending on the cognitive task, i.e. the VFT-1 or the Stroop test, in both genders. The correlations of the Oxy-Hb increase during the cognitive tasks with the BDNF genotype and serum BDNF level were negative. Our data suggest that the temporal mental status might affect the course of the prefrontal $\mathrm{Oxy}-\mathrm{Hb}$ change during certain cognitive tasks and that NIRS measurement has the potential to be a useful tool for detecting early subclinical manifestations of depressed mood in males that are not correlated with the individual properties of BDNF.

\section{ACKNOWLEDGEMENTS}

A part of this research was supported by the Strategic Research Program for Brain Sciences "Brain Machine Interface Development" by the Ministry of Education, Culture, Sports, Science and Technology of Japan.

\section{REFERENCES}

[1] Adler, D.A., McLaughlin, T.J., Rogers, W.H., Chang, H., Lapitsky, L. and Lerner, D. (2006) Job performance deficits due to depression. American Journal of Psychiatry, 163, 1569-1576. doi:/10.1176/appi.ajp.163.9.1569

[2] Kessler, R.C., Berglund, P., Demler, O., Jin, R., Merikangas, K.R. and Walters, E.E. (2005) Lifetime prevalence and age-of-onset distributions of DSM-IV disorders in the National Comorbidity Survey Replication. Archives of General Psychiatry, 62, 593-602. 
[3] Pignone, M.P., Gaynes, B.N., Rushton, J.L., Burchell, C. M., Orleans, C.T., Mulrow, C.D. and Lohr, K.N. (2002) Screening for depression in adults: A summary of the evidence for the U.S. Preventive Services Task Force. Annals of Internal Medicine, 136, 765-776.

[4] Hammen, C., Kim, E.Y., Eberhart, N.K. and Brennan, P.A. (2009) Chronic and acute stress and the prediction of major depression in women. Depression and Anxiety, 26, 718-723. doi:/10.1002/da.20571

[5] Mazure, C. (1998) Life Stressors as risk factors in depression. Clinical Psychology: Science and Practice, 5, 291-313.

[6] Maki, A., Yamashita, Y., Ito, Y., Watanabe, E., Mayanagi, Y. and Koizumi, H. (1995) Spatial and temporal analysis of human motor activity using noninvasive NIR topography. Medical Physics, 22, 1997-2005. doi:/10.1118/1.597496

[7] Irani, F., Platek, S.M., Bunce, S., Ruocco, A.C. and Chute, D. (2007) Functional near infrared spectroscopy (fNIRS): An emerging neuroimaging technology with important applications for the study of brain disorders. Clinical Neuropsychologist, 21, 9-37.

doi:/10.1080/13854040600910018

[8] Herrmann, M.J., Ehlis, A.C. and Fallgatter, A.J. (2004) Bilaterally reduced frontal activation during a verbal fluency task in depressed patients as measured by near-infrared spectroscopy. Journal of Neuropsychiatry \& Clinical Neurosciences, 16, 170-175. doi:/10.1176/appi.neuropsych.16.2.170,

[9] Kameyama, M., Fukuda, M., Yamagishi, Y., Sato, T., Uehara, T., Ito, M., Suto, T. and Mikuni, M. (2006) Frontal lobe function in bipolar disorder: A multichannel near-infrared spectroscopy study. Neuroimage, 29, 172184. doi:/10.1016/j.neuroimage.2005.07.025

[10] Suto, T., Fukuda, M., Ito, M., Uehara, T. and Mikuni, M. (2004) Multichannel near-infrared spectroscopy in depression and schizophrenia: Cognitive brain activation study. Biological Psychiatry, 55, 501-511. doi:/10.1016/j.biopsych.2003.09.008

[11] Marcus, S.M., Young, E.A., Kerber, K.B., Kornstein, S., Farabaugh, A.H., Mitchell, J., Wisniewski, S.R., Balasubramani, G.K., Trivedi, M.H. and Rush, A.J. (2005) Gender differences in depression: Findings from the STAR*D study. Journal of Affective Disorders, 87, 141150. doi:/10.1016/j.jad.2004.09.008

[12] Binder, D.K. and Scharfman, H.E. (2004) Brain-derived neurotrophic factor. Growth Factors, 22, 123-131. doi:/10.1080/08977190410001723308

[13] Hariri, A.R., Goldberg, T.E., Mattay, V.S., Kolachana, B.S., Callicott, J.H., Egan, M.F. and Weinberger, D.R. (2003) Brain-derived neurotrophic factor val66met polymorphism affects human memory-related hippocampal activity and predicts memory performance. Journal of Neuroscience, 23, 6690-6694.

[14] Egan, M.F., Kojima, M., Callicott, J.H., Goldberg, T.E., Kolachana, B.S., Bertolino, A., Zaitsev, E., Gold, B., Goldman, D., Dean, M., Lu, B. and Weinberger, D.R. (2003) The BDNF val66met polymorphism affects activity-dependent secretion of BDNF and human memory and hippocampal function. Cell, 112, 257-269. doi:/10.1016/S0092-8674(03)00035-7

[15] Dempster, E., Toulopoulou, T., McDonald, C., Bramon, E., Walshe, M., Filbey, F., Wickham, H., Sham, P.C., Murray, R.M. and Collier, D.A. (2005) Association between BDNF val66 met genotype and episodic memory. American Journal of Medical Genetics Part B: Neuropsychiatric Genetics, 134B, 73-75. doi:/10.1002/ajmg.b.30150

[16] Rybakowski, J.K., Borkowska, A., Czerski, P.M., Skibinska, M. and Hauser, J. (2003) Polymorphism of the brain-derived neurotrophic factor gene and performance on a cognitive prefrontal test in bipolar patients. Bipolar Disorder, 5, 468-472. doi:/10.1046/j.1399-5618.2003.00071.x

[17] Karege, F., Bondolfi, G., Gervasoni, N., Schwald, M., Aubry, J.M. and Bertschy, G. (2005) Low brain-derived neurotrophic factor (BDNF) levels in serum of depressed patients probably results from lowered platelet BDNF release unrelated to platelet reactivity. Biological Psychiatry, 57, 1068-1072. doi:/10.1016/j.biopsych.2005.01.008

[18] Rybakowski, J.K., Borkowska, A., Skibinska, M. and Hauser, J. (2006) Illness-specific association of val66met BDNF polymorphism with performance on Wisconsin Card Sorting Test in bipolar mood disorder. Molecular Psychiatry, 11, 122-124. doi:/10.1038/sj.mp.4001765

[19] Gonul, A.S., Akdeniz, F., Taneli, F., Donat, O., Eker, C. and Vahip, S. (2005) Effect of treatment on serum brainderived neurotrophic factor levels in depressed patients. European Archives of Psychiatry and Clinical Neuroscience, 255, 381-386. doi:/10.1007/s00406-005-0578-6

[20] Shimizu, E., Hashimoto, K., Okamura, N., Koike, K., Komatsu, N., Kumakiri, C., Nakazato, M., Watanabe, H., Shinoda, N., Okada, S. and Iyo, M. (2003) Alterations of serum levels of brain-derived neurotrophic factor (BDNF) in depressed patients with or without antidepressants. Biological Psychiatry, 54, 70-75. doi:/10.1016/S0006-3223(03)00181-1

[21] Lang, U.E., Hellweg, R. and Gallinat, J. (2004) BDNF serum concentrations in healthy volunteers are associated with depression-related personality traits. Neuropsychopharmacology, 29, 795-798. doi:/10.1038/sj.npp.1300382

[22] Zigmond, A.S. and Snaith, R.P. (1983) The hospital anxiety and depression scale. Acta Psychiatrica Scandinavica, 67, 361-370. doi:/10.1111/j.1600-0447.1983.tb09716.x

[23] Association, A.P. (1994) Diagnostic and statistical manual of mental disorders. 4th Edition, American Psychiatric Press, Washington DC.

[24] Watanabe, E., Maki, A., Kawaguchi, F., Takashiro, K., Yamashita, Y., Koizumi, H. and Mayanagi, Y. (1998) Non-invasive assessment of language dominance with near-infrared spectroscopic mapping. Neuroscience Letters, 256, 49-52. doi:/10.1016/S0304-3940(98)00754-X

[25] MacLeod, C.M. (1991) Half a century of research on the Stroop effect: An integrative review. Psychological Bulletin, 109, 163-203. doi:/10.1037/0033-2909.109.2.163

[26] Shimada, S. and Hiraki, K. (2006) Infant's brain re- 
sponses to live and televised action. Neuroimage, 32, 930-939. doi:/10.1016/j.neuroimage.2006.03.044

[27] Minagawa-Kawai, Y., Mori, K., Furuya, I., Hayashi, R. and Sato, Y. (2002) Assessing cerebral representations of short and long vowel categories by NIRS. Neuroreport, 13, 581-584.

[28] Shimoda, N., Takeda, K., Imai, I., Kaneko, J. and Kato, H. (2008) Cerebral laterality differences in handedness: A mental rotation study with NIRS. Neuroscience Letters, 430, 43-47. doi:/10.1016/i.neulet.2007.10.016

[29] Takeda, K., Gomi, Y., Imai, I., Shimoda, N., Hiwatari, M. and Kato, H. (2007) Shift of motor activation areas during recovery from hemiparesis after cerebral infarction: A longitudinal study with near-infrared spectroscopy. Neuroscience Research, 59, 136-144. doi:/10.1016/j.neures.2007.06.1466,

[30] Itoh, K., Hashimoto, K., Kumakiri, C., Shimizu, E. and Iyo, M. (2004) Association between brain-derived neurotrophic factor $196 \mathrm{G} / \mathrm{A}$ polymorphism and personality traits in healthy subjects. American Journal of Medical Genetics Part B: Neuropsychiatric Genetics, 124B, 61-63. doi:/10.1002/ajmg.b.20078

[31] Kakimoto, Y., Nishimura, Y., Hara, N., Okada, M., Tanii, H. and Okazaki, Y. (2009) Intrasubject reproducibility of prefrontal cortex activities during a verbal fluency task over two repeated sessions using multi-channel near-infrared spectroscopy. Psychiatry and Clinical Neurosciences, 63, 491-499. doi:/10.1111/j.1440-1819.2009.01988.x

[32] Kono, T., Matsuo, K., Tsunashima, K., Kasai, K., Takizawa, R., Rogers, M.A., Yamasue, H., Yano, T., Taketani, Y. and Kato, N. (2007) Multiple-time replicability of near-infrared spectroscopy recording during prefrontal activation task in healthy men. Neuroscience Research, 57, 504-512. doi:/10.1016/j.neures.2006.12.007

[33] Braver, T.S., Cohen, J.D., Nystrom, L.E., Jonides, J., Smith, E.E. and Noll, D.C. (1997) A parametric study of prefrontal cortex involvement in human working memory. Neuroimage, 5, 49-62. doi:/10.1006/nimg.1996.0247

[34] Fridriksson, J. and Morrow, L. (2005) Cortical activation and language task difficulty in aphasia. Aphasiology, 19, 239-250. doi:/10.1080/02687030444000714

[35] Drevets, W.C. (2000) Neuroimaging studies of mood disorders. Biological Psychiatry, 48, 813-829. doi:/10.1016/S0006-3223(00)01020-9

[36] Galynker, II, Cai, J., Ongseng, F., Finestone, H., Dutta, E. and Serseni, D. (1998) Hypofrontality and negative symptoms in major depressive disorder. Journal of Nuclear Medicine, 39, 608-612.

[37] Milak, M.S., Parsey, R.V., Keilp, J., Oquendo, M.A., Malone, K.M. and Mann, J.J. (2005) Neuroanatomic correlates of psychopathologic components of major depressive disorder. Archives of General Psychiatry, 62, 397408. doi:/10.1001/archpsyc.62.4.397

[38] Kameyama, M., Fukuda, M., Uehara, T. and Mikuni, M. (2004) Sex and age dependencies of cerebral blood volume changes during cognitive activation: A multichannel near-infrared spectroscopy study. Neuroimage, 22, 1715-

\section{1. doi:/10.1016/j.neuroimage.2004.03.050}

[39] Herrmann, M.J., Walter, A., Ehlis, A.C. and Fallgatter, A.J. (2006) Cerebral oxygenation changes in the prefrontal cortex: Effects of age and gender. Neurobiology of Aging, 27, 888-894. doi:/10.1016/j.neurobiolaging.2005.04.013

[40] Ehlis, A.C., Herrmann, M.J., Wagener, A. and Fallgatter, A.J. (2005) Multi-channel near-infrared spectroscopy detects specific inferior-frontal activation during incongruent Stroop trials. Biological Psychology, 69, 315-331. doi:/10.1016/j.biopsycho.2004.09.003

[41] Schroeter, M.L., Zysset, S., Kruggel, F. and Von Cramon, D.Y. (2003) Age dependency of the hemodynamic response as measured by functional near-infrared spectroscopy. Neuroimage, 19, 555-564. doi:/10.1016/S1053-8119(03)00155-1

[42] Yanagisawa, H., Dan, I., Tsuzuki, D., Kato, M., Okamoto, M., Kyutoku, Y. and Soya, H. (2010) Acute moderate exercise elicits increased dorsolateral prefrontal activation and improves cognitive performance with Stroop test. Neuroimage, 50, 1702-1710. doi:/10.1016/j.neuroimage.2009.12.023

[43] Kerns, J.G., Cohen, J.D., MacDonald, A.W., Johnson, M.K., Stenger, V.A., Aizenstein, H. and Carter, C.S. (2005) Decreased conflict- and error-related activity in the anterior cingulate cortex in subjects with schizophrenia. American Journal of Psychiatry, 162, 1833-1839. doi:/10.1176/appi.ajp.162.10.1833

[44] Yucel, M., Pantelis, C., Stuart, G.W., Wood, S.J., Maruff, P., Velakoulis, D., Pipingas, A., Crowe, S.F., TochonDanguy, H.J. and Egan, G.F. (2002) Anterior cingulate activation during Stroop task performance: A PET to MRI coregistration study of individual patients with schizophrenia. American Journal of Psychiatry, 159, 251254. doi:/10.1176/appi.ajp.159.2.251

[45] Gur, R.C., Gur, R.E., Obrist, W.D., Hungerbuhler, J.P., Younkin, D., Rosen, A.D., Skolnick, B.E. and Reivich, M. (1982) Sex and handedness differences in cerebral blood flow during rest and cognitive activity. Science, 217, 659661. doi:/10.1126/science.7089587

[46] Rodriguez, G., Warkentin, S., Risberg, J. and Rosadini, G. (1988) Sex differences in regional cerebral blood flow. Journal of Cerebral Blood Flow \& Metabolism, 8, 783789. doi:/10.1038/jcbfm.1988.133

[47] Kansaku, K., Yamaura, A. and Kitazawa, S. (2000) Sex differences in lateralization revealed in the posterior language areas. Cerebral Cortex, 10, 866-872. doi:/10.1093/cercor/10.9.866

[48] Hariri, A.R., Goldberg, T.E., Mattay, V.S., Kolachana, B.S., Callicott, J.H., Egan, M.F. and Weinberger, D.R. (2003) Brain-derived neurotrophic factor val66met polymorphism affects human memory-related hippocampal activity and predicts memory performance. Journal of Neuroscience, 23, 6690-6694.

[49] Rybakowski, J.K., Borkowska, A., Skibinska, M. and Hauser, J. (2006) Illness-specific association of val66met BDNF polymorphism with performance on Wisconsin Card Sorting Test in bipolar mood disorder. Molecular Psychiatry, 11, 122-124. doi:/10.1038/sj.mp.4001765 
[50] Shimizu, E., Hashimoto, K. and Iyo, M. (2004) Ethnic difference of the BDNF 196G/A (val66met) olymorphism frequencies: The possibility to explain ethnic mental traits. American Journal of Medical Genetics Part B: Neuropsychiatric Genetics, 126B, 122-123.

doi:/10.1002/ajmg.b.20118
[51] Takizawa, R., Tochigi, M., Kawakubo, Y., Marumo, K., Sasaki, T., Fukuda, M. and Kasai, K. (2009) Association between catechol-O-methyltrasferase Val108/158Met genotype and prefrontal hemodynamic response in schizophrenia. PLoS One, 4, e5495.

doi:/10.1371/journal.pone.0005495 